\title{
C-S cross-coupling of aryl halides with alkyl thiols catalyzed by in-situ generated nickel(II) $\mathbf{N}$-heterocyclic carbene complexes
}

\author{
Fang-Jie Guo, ${ }^{\mathrm{a}}$ Jing Sun, ${ }^{\mathrm{a}, *}$ Zhao-Qing Xu, ${ }^{\mathrm{b}}$ Fritz E. Kühn, ${ }^{\mathrm{c}}$ Shu-Liang Zang, ${ }^{\mathrm{a}}$ and \\ Ming-Dong Zhou ${ }^{\mathrm{a}, *}$
}

\begin{abstract}
The C-S cross-coupling of aryl halides with alkyl thiols catalyzed by in-situ generated Ni (II) N-heterocyclic carbene (NHC) complexes is investigated. Good to excellent yields can be obtained for a variety of aryl halides when using 5 mol\% of the $\mathrm{Ni}$ (II)-NHC catalyst and 1.5 eq. of $\mathrm{KO}^{\mathrm{t}} \mathrm{Bu}$. Both the electronic and steric effects of the NHC ligands on the catalytic performance of $\mathrm{Ni}$ (II)-NHC, as well as the electronic effects of aryl halides on coupling reactivity are examined. The mechanism for Ni (II)-NHC catalyzed coupling reactions is also discussed.
\end{abstract}

Keywords: C-S cross coupling; Nickel catalysis; N-heterocyclic carbene; Aryl sulfides

\section{Introduction}

C-S coupling is a very important transformation in modern organic synthesis. Catalytic cross-coupling of aryl halide and thiol provides a simple and efficient protocol for the synthesis of aryl sulfides. The latter are important synthetic intermediates in biochemistry and in pharmaceutical industry. ${ }^{1,2}$

Among various reported procedures, palladium ${ }^{3,4}$ and copper $^{5-7}$ based catalysts are 
most commonly used under basic conditions. In addition, nickel, ${ }^{8-11}$ iron, ${ }^{12}$ indium ${ }^{13,14}$ and other metal compounds ${ }^{15-18}$ are also found to be effective catalysts. However, in contrast to the large number of reports on palladium catalysts, the C-S coupling catalyzed by nickel and other transition metal complexes is so far rather limited in scope. Since nickel is cheaper and also shows a reduced tendency to deposit metallic nanoparticles (as compared to $\mathrm{Pd}$ ), ${ }^{8-11}$ the C-S cross-coupling using $\mathrm{Ni}$ complex appears to be an attractive field of study.

Additionally, N-heterocyclic carbenes (NHCs) have received much attention as ligands in transition-metal catalysis during the last two decades. ${ }^{19-25} \mathrm{Ni}-\mathrm{NHC}$ complexes, for example, are known to be excellent catalysts for numerous transformations such as Kumada-Corriu cross-coupling ${ }^{26}$, Suzuki-Miyaura cross-coupling $^{27}$, hydrosilylation reactions ${ }^{28}$, the amination of arylamines ${ }^{29}$, and the oligomerization of olefins. ${ }^{30}$ However, to the best of our knowledge, the C-S cross-coupling using Ni-NHC complexes, has not yet been extensively studied. ${ }^{31-33}$ Zhang et. al. have described a Ni (0)-NHC catalyzed C-S coupling using 1,3-dibenzylimidazol-2-ylidene as the NHC precursor, ${ }^{31}$ and good to excellent yields could be achieved for a variety of aryl halides. However, in this reaction protocol the coupling of aryl halides with alkyl thiols were not investigated. ${ }^{31}$ Considering that alkyl phenyl thioethers are also important synthetic intermediates, and Ni (II)-NHC complexes may represent better stability in the catalytic system than $\mathrm{Ni}(0)$ complexes, we were interested in studying the C-S cross-coupling of aryl halides with alkyl thiols using in-situ generated Ni (II)-NHC complexes as the catalysts. Therefore, we wish to report our primary results of this work herein.

\section{Results and discussion}

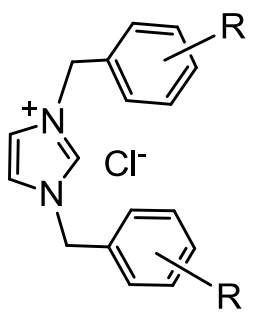

$\mathrm{R}=\mathrm{H}(\mathrm{L} 1) ; \mathrm{R}=4-\mathrm{CH}_{3}$ (L2); R= 4-

$\mathrm{OCH}_{3}(\mathrm{~L} 3) ; \mathrm{R}=4-\mathrm{Cl}(\mathrm{L} 4) ; \mathrm{R}=2,6-\mathrm{Cl}$

(L5); $\mathrm{R}=4{ }^{-\mathrm{t}} \mathrm{Bu}$ (L6)

Scheme 1. NHC ligand precursors. 
NHCs L1-L6 were investigated as ligands for Ni catalysts in C-S coupling reactions (Scheme 1). In the presence of $\mathrm{KO}^{\mathrm{t}} \mathrm{Bu}$ as deprotonating reagent, L1-L6 readily coordinate with $\mathrm{NiCl}_{2}$ forming the corresponding nickel bis-carbene complexes, which can be proven by the isolated X-ray single crystal structures of Ni(II)-NHCs bearing L1, L2 and L4 (Figure 1). Details on the synthesis, crystallization procedures and characterization data of $\mathrm{Ni}(\mathrm{II})-\mathrm{NHCs}$ can be found in the ESI.
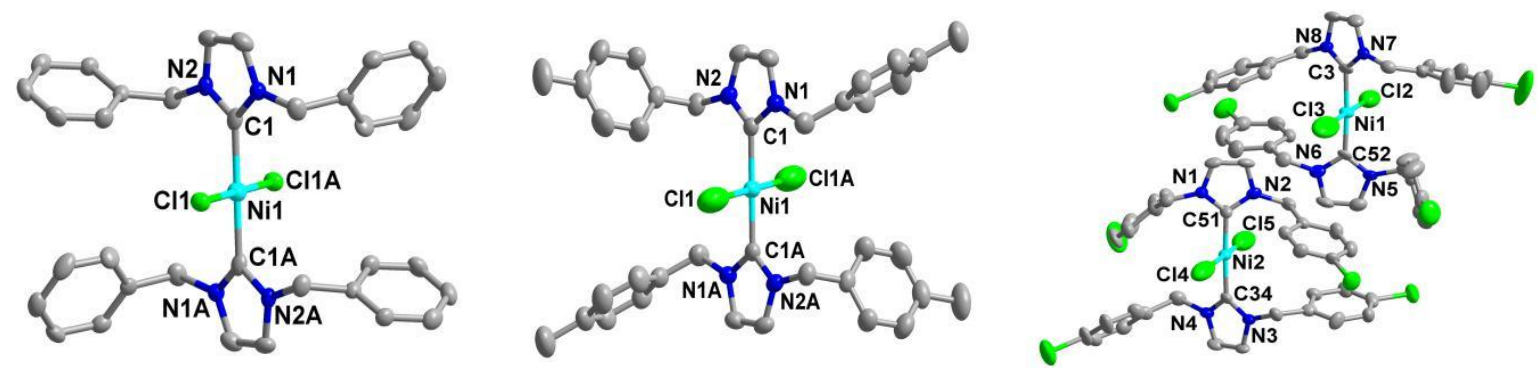

Figure 1. ORTEP diagram showing the coordination environment for Ni atoms of Ni-NHCs.

Thermal ellipsoids are given on a 50\% probability level. H-atoms have been omitted. CCDC: 1433176 (L1), 1433177 (L2), 1433179 (L4).

To explore the reactivity, coupling of 4-bromotoluene with butanethiol was studied first (Table 1). Blank reaction resulted in only trace amounts of butyl(4-methylphenyl)sulfane 3aa in the absence of catalyst. When using 5 mol\% of $\mathrm{NiCl}_{2}$ without involving a NHC ligand, $35 \%$ of 3aa could be obtained (entry 1). After applying $10 \mathrm{~mol} \%$ NHC ligands L1-L6, the reaction readily formed the respective nickel bis-carbene complexes. As compared to $\mathrm{NiCl}_{2}$, the in-situ generated $\mathrm{Ni}$ (II)-NHC compounds showed much better solubility in the reaction system. However, different ligand precursors displayed very different catalytic activities (entries 2-7). It seems that both electronic and steric effects of the NHC ligands played important roles on catalytic performance. For $\mathbf{L 2}$ and $\mathbf{L 3}$ bearing strong electron-donating $p-\mathrm{CH}_{3}$ and $p-\mathrm{OCH}_{3}$ groups on the benzene ring, the yields of 3aa were lower than that using merely $\mathrm{NiCl}_{2}$ (entries 3 and 4). This may be due to the strong electron-donating ability of $\mathbf{L 2}$ and $\mathbf{L 3}$, leading to a strong decrease of Lewis acidity of the Ni center. For $\mathrm{Ni}$ (II)-NHC L1 without a functional group on the NHC ligand, the reaction showed a comparable 3aa yield as when using $\mathrm{NiCl}_{2}$ (entry 2). $\mathrm{Ni}$ (II)-NHC (L4) with an 
electron-withdrawing $p$-Cl group resulted in the highest 3aa yield (53\%, entry 5), and $\mathrm{Ni}$ (II)-NHC(L5) with two $\mathrm{Cl}$ groups led to $50 \%$ of 3aa (entry 6). Owing to both the strong electron-donating ability and steric hindrance of a tert-butyl group, only $20 \%$ of 3aa was obtained when using Ni(II)-NHC (L6) (entry 7). When reducing the catalyst amount from 5 to $3 \mathrm{~mol} \%$, the product yield was also significantly decreased (entry 8 ). The reaction was further optimized by using different solvents (entries 5, 9, 10). DMF proved to be the most favorable solvent.

Table 1. C-S cross-coupling of 4-bromotoluene with butanethiol catalyzed by Ni (II)-NHCs. ${ }^{\text {a }}$

\begin{tabular}{ccccc}
2 & & & \\
\hline
\end{tabular}


Coupling of chlorobenzene with butanethiol gave $40 \%$ of butylphenylthioether 3ak, a lower yield than that obtained when using bromobenzene $(63 \%)$.

Table 2.C-S cross-coupling of aryl halides with thiols catalyzed by Ni (II)-NHC (L4). ${ }^{\text {a,b }}$

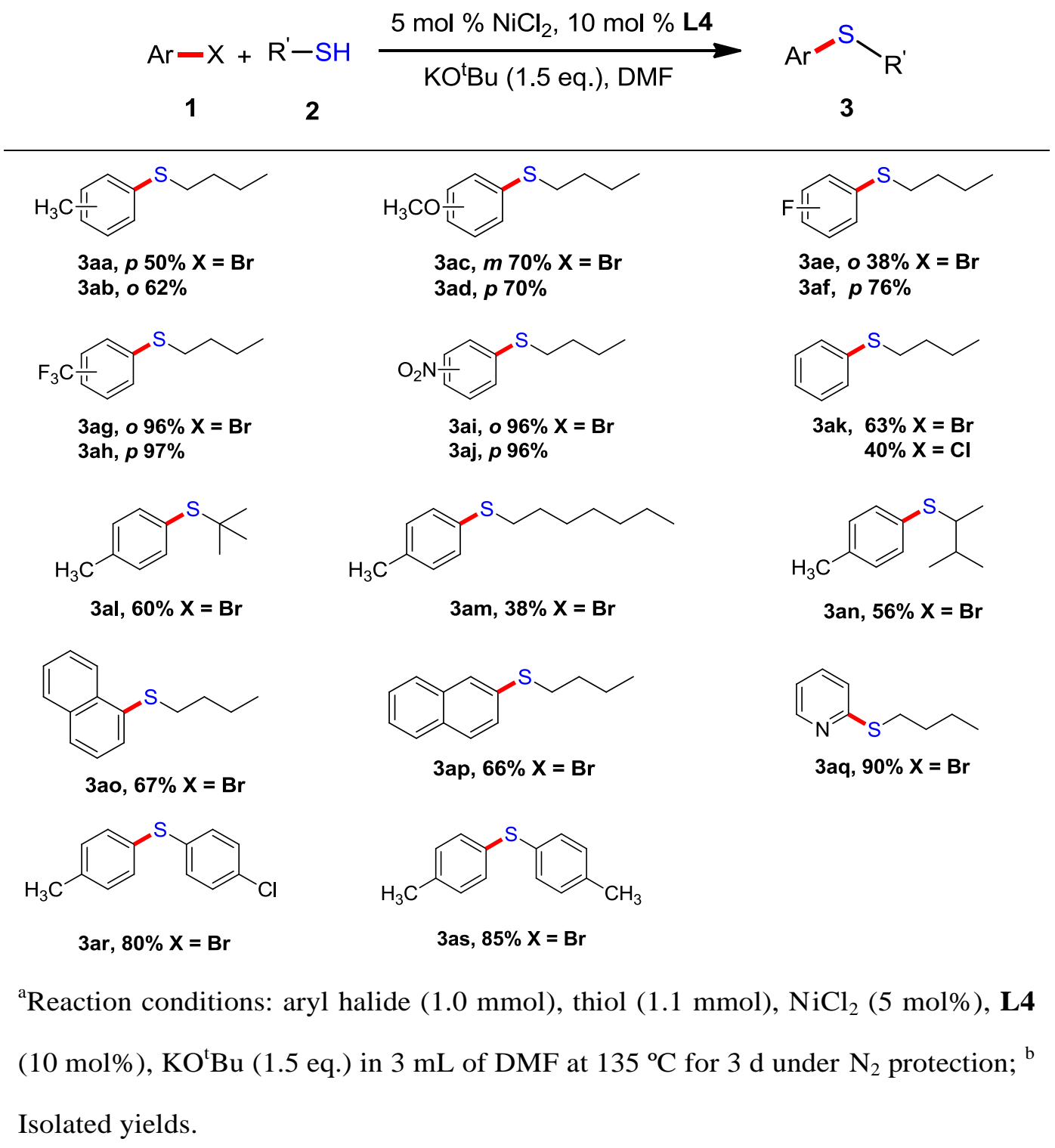

Good thioether yields were also achieved for bromonaphthalenes and pyridine bromide under optimal conditions. Moreover, the cross-coupling proved to be easier when aryl thiol is used instead of alkyl thiol. It can be seen that coupling of $p$-methyl bromobenzene with para-chloro or methyl substituted thiolphenol affords $80 \%$ and $85 \%$ of product yield respectively.

\section{Mechanistic considerations}


Based on literature precedents ${ }^{8-11,31-33}$ and experimental observations, a possible mechanism for the Ni (II)-NHC catalyzed C-S cross-coupling is proposed in Scheme 2. First, a ligand exchange may occur between RS and $\mathrm{X}$ of the Ni (II)-NHC catalyst $\mathbf{A}$, forming an intermediate $\mathbf{B}$. B undergoes reductive elimination to form a $\mathrm{Ni}(0)$ species, which is assumed to be the real active species in catalysis. The observation of $13 \%$ of 1, 2-dibutyldisulfane gave solid evidence for such an assumption (Table 1, entry 5). Subsequent oxidative addition of ArX to $\mathbf{C}$ affords an intermediate $\mathbf{D}$. Further ligand exchanging of $\mathbf{D}$ with RSH leads to the formation of an intermediate $\mathbf{E}$. $\mathbf{E}$ undergoes reductive elimination and transfers back to $\mathbf{C}$, forming the desired thioether product.

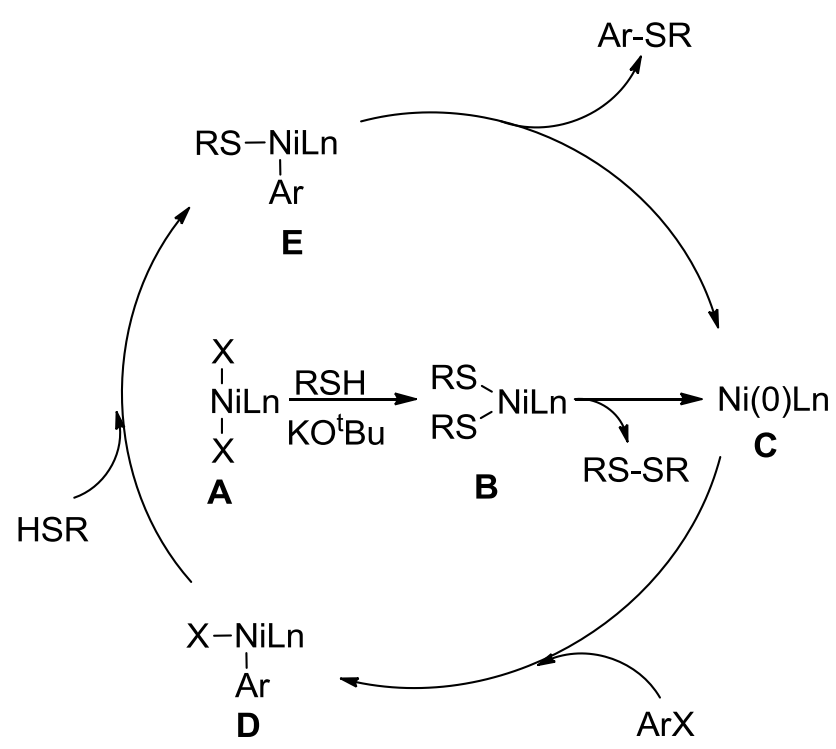

Scheme 2. Possible mechanism for the Ni (II)-NHC catalyzed C-S cross-coupling.

\section{Experimental Section}

General procedure for the coupling reaction. In a typical procedure, $\mathrm{NiCl}_{2}(6.5 \mathrm{mg}, 0.05$ mmol), NHC ligand precursor $\mathbf{L 4}$ (35 mg, $0.1 \mathrm{mmol}), \mathrm{KO}^{\mathrm{t}} \mathrm{Bu}(168 \mathrm{mg}, 1.5 \mathrm{mmol})$ were added to a Schlenk tube under an atmosphere of nitrogen, DMF $(3 \mathrm{~mL})$ was then injected and stirred. After 30 minutes, aryl halide $(1.0 \mathrm{mmol})$ and thiol $(1.1 \mathrm{mmol})$ were injected, and the mixture was immediately heated to $135^{\circ} \mathrm{C}$ under vigorous stirring. After the reaction, the mixture was cooled to room temperature and quenched by addition of distilled water. The aqueous phase was extracted with ethyl acetate for three times. The combined organic layers were dried with $\mathrm{MgSO}_{4}$, and the sample was used for GC analysis. The sample solution was further 
evaporated under vacuum to remove the solvent. The obtained crude product was purified by column chromatography (eluent, petroleum) on silica gel to afford the desired thioethers.

\section{Conclusions}

In conclusion, C-S cross-coupling of aryl halides with alkyl thiols catalyzed by in-situ generated Ni (II)-NHCs is described. The examined Ni (II)-NHC complexes are efficient catalysts for the coupling of various aryl halides. Both electronic and steric effects of functional groups on the aromatic ring of the NHC ligands affect the catalytic performance of $\mathrm{Ni}$ (II)-NHCs significantly. In the coupling reaction, a reductive elimination of $\mathrm{Ni}$ (II)-NHC complex may initially occur, forming an $\mathrm{Ni}(0)$ species, which is assumed to be the real active species. Similar to other nickel or palladium catalyzed coupling reactions, the active $\mathrm{Ni}(0)$ species may further undergo oxidative addition with ArX, the ligand exchanges of Ar-Ni-X with RSH, and finally reductive elimination of Ar-Ni-SR to form the desired Ar-SR coupling product.

\section{Acknowledgements}

M.D.Z thanks the National Science Foundation of China (21101085), Natural Science Foundation of Liaoning Province (2015020196), Fushun Science \& Technology Program (FSKJHT 201423), Liaoning Excellent Talents Program in University (LJQ2012031), and Talent Scientific Research Fund of LSHU (No. 2016XJJ-006) for the financial supports.

\section{Notes and references}

1 I. P. Beletskaya, V. P. Ananikov, Chem. Rev., 2011, 111, 1596-636.

2 H. Liu, X. Jiang. Chem. Asian J., 2013, 8, 2546-2563.

3 A. Byeun, K. Baek, M. S. Han, S. Lee, Tetrahedron Lett., 2013, 54, 6712-6715.

4 E. Alvaro, J. F. Hartwig, J. Am. Chem. Soc., 2009, 131, 7858-7868.

5 F. Luo, C. Pan, L. P. Li, F. Chen, J. Cheng, Chem. Commun., 2011, 47, 5304-5306.

6 S. Mallick, S. Rana, K. Parida, Dalton Trans., 2011, 40, 9169-9175.

7 C. F. Lee, Y. C. Liu, S. S. Badsara, Chem. Asian J., 2014, 9, 706-722. 
8 J. Zhang, C. M. Medley, J. A. Krause, H. Guan, Organometallics, 2010, 29, 6393-6401.

9 G. T. Venkanna, H. D. Arman, Z. J. Tonzetich, ACS Catal., 2014, 4, 2941-2950.

10 S. Jammi, P. Barua, L. Rout, P. Saha, T. Punniyamurthy, Tetrahedron Lett., 2008, 49, 1484-1487.

11 O. Baldovino-Pantaleón, S. Hernández-Ortega, D. Morales-Morales, Adv. Syn. Catal., 2006, 348, 236-242.

12 A. Correa, M. Carril, C. Bolm, Angew. Chem. Int. Ed., 2008, 47, 2880-2883.

13 V. P. Reddy, A. V. Kumar, K. Swapna, K. R. Rao, Org. Lett., 2009, 11, 1697-1700.

14 V. P. Reddy, K. Swapna, A. V. Kumar, K. R. Rao, J. Org. Chem., 2009, 74, 3189-3191.

15 S. N. Murthy, B. Madhav, V. P. Reddy, Y. V. D. Nageswar, Eur. J. Org. Chem., 2009, 34, 5902-5905.

16 Y. Wong, T. T. Jayanth, C. Cheng, Org. Lett., 2006, 8, 5613-5616.

17 M. Lan, W. Wu, S. Huang, K. Luo, F. Tsai, RSC. Adv., 2011, 1, 1751-1755.

18 C. Lai, H. Kao, Y. Wang, C. Lee, Tetrahedron Lett., 2012, 53, 4365-4367.

19 W. A. Herrmann, C. Köcher, Angew. Chem. Int. Ed., 1997, 36, 2162-2187.

20 W. A. Herrmann, Angew. Chem. Int. Ed., 2002, 41, 1290-1309.

21 F. E. Hahn, Angew. Chem. Int. Ed.,2006, 45, 1348-1352.

22 K. Riener, S. Haslinger, A. Raba, M. P. Högerl, M. Cokoja, W. A. Herrmann, F. E. Kühn, Chem. Rev., 2014, 114, 5215-5272.

23 G. F. Du, H. Guo, Y. Wang, W. J. Li , W. J. Shi, B. Dai, J. Saudi Chem. Soc., 2015, 19, 112-115.

24 G. F. Du, X. L. Zou, B. Dai, L. He, J. Saudi Chem. Soc., 2016, 20, 207-212.

25 L. A. Schaper, S. J. Hock, W. A. Herrmann and F. E. Kühn, Angew. Chem. Int. Ed., 2013, 52, 270-289.

26 Z. Xi, B. Liu, W. Chen, J. Org. Chem., 2008, 73, 3954-3957.

27 Z. Xi, X. Zhang, W. Chen, S. Fu, D. Wang, Organometallics, 2007, 26, 6636-6642. 28 L. P. Bheeter, M. Henrion, L. Brelot, C. Darcel, M. J. Chetcuti, J. B. Sortais, V. Ritleng, Adv. Synth. \& Catal., 2012, 354, 2619-2624. 
29 A. R. Martin, Y. Makida, S. Meiries, A. M. Z. Slawin, S. P. Nolan, Organometallics, 2013, 32, 6265-6270.

30 W. Buchowicz, A. Kozioł, L. B. Jerzykiewicz, T. Lis, S. Pasynkiewicz, A. Pecherzewska, A. Pietrzykowski, J. Mol. Catal. A: Chem., 2006, 257, 118-123.

31 Y. G. Zhang, K. C. Ngeow, J. Y. Ying, Org. Lett., 2007, 9, 3495-3498.

32 P. Guan, C. Cao, Y. Liu, Y. Li, P. He, Q. Chen, G. Liu, Y. Shi, Tetrahedron Lett., $2012, \mathbf{5 3}, 5987-5992$.

33 A. R. Martin, D. J. Nelson, S. Meiries, A. M. Z. Slawin, S. P. Nolan, Eur. J. Org. Chem., 2014, 15, 3127-3131. 


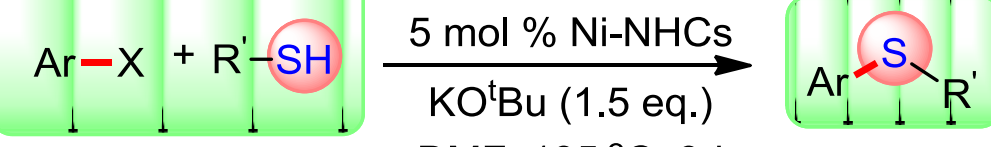

$$
\begin{aligned}
& \text { DMF, } 135^{\circ} \mathrm{C}, 3 d
\end{aligned}
$$

Nickel (II) N-heterocyclic carbene complex has been applied as efficient catalyst for the C-S cross-coupling of aryl halides with alkyl thiols, affording various alkylphenyl thioethers as the coupling products. 